\title{
Healthy fats
}

\section{The Queen of Fats: Why Omega-3s Were \\ Removed from the Western Diet and What We Can Do to Replace Them by Susan Allport \\ University of California Press: 2006. \\ 232 pp. \$22.50, £14.95}

\section{Asim K. Duttaroy}

Two series of polyunsaturated fatty acids, the omega- 6 and the omega- 3 fatty acids, are essential dietary nutrients. Two major omega-3 fatty acids, eicosa-pentaenoic acid (EPA) and docosa-hexaenoic acid (DHA), are increasingly being seen as important modulators of biological pathways that affect growth, development and health. Consumption of these omega- 3 fatty acids, which are found in fatty fish and fish oils, has been linked to the low incidence of coronary heart disease in the Inuit people of Greenland. An omega-3 fatty acid with a shorter chain, alpha-linolenic acid (ALA), found in some plant oils and sometimes converted to EPA and DHA, may also be beneficial. Omega- 3 fatty acids may protect against cardiovascular disease by several mechanisms, such as lowering blood pressure; reducing plasma triglycerides; lowering the platelet aggregation response, inflammation and arrhythmias; and improving endothelial function, insulin sensitivity and atherosclerotic plaque stability.

Susan Allport discusses the history of using omega-3 fatty acids in her well-written book The Queen of Fats. She has done an excellent job in describing the events and the international collaboration involved in discovering the effects of omega- 3 fatty acids. She covers almost every aspect of omega- $3 \mathrm{~s}$, including the importance of an appropriate ratio of omega- 6 and omega- 3 fatty acids in the diet, which has been biased towards omega- 6 fatty acids over the past 50 years, and the consequences for our health of changes in the ratio. There is considerable controversy over how much omega- 6 and omega- 3 fatty acids are needed in the diet, and which ones.

Many studies have shown a correlation between omega -3 fatty acids and diseases, but translating these findings into recommended amounts for individuals and populations requires careful consideration. Meta-analyses of the effects of omega- 3 fatty acids are not conclusive on total mortality, combined cardiovascular events, or cancer. However, some of these meta-analyses failed to take into account many of the pitfalls that must be addressed if a clear picture of the benefits of omega- 3 fatty acids is to be obtained.

Allport does not discuss recent meta-analyses of several large studies on fish oils, and fails to mention some of the possible health risks associated with a high intake of fish oils, such as increased bleeding and haemorrhagic

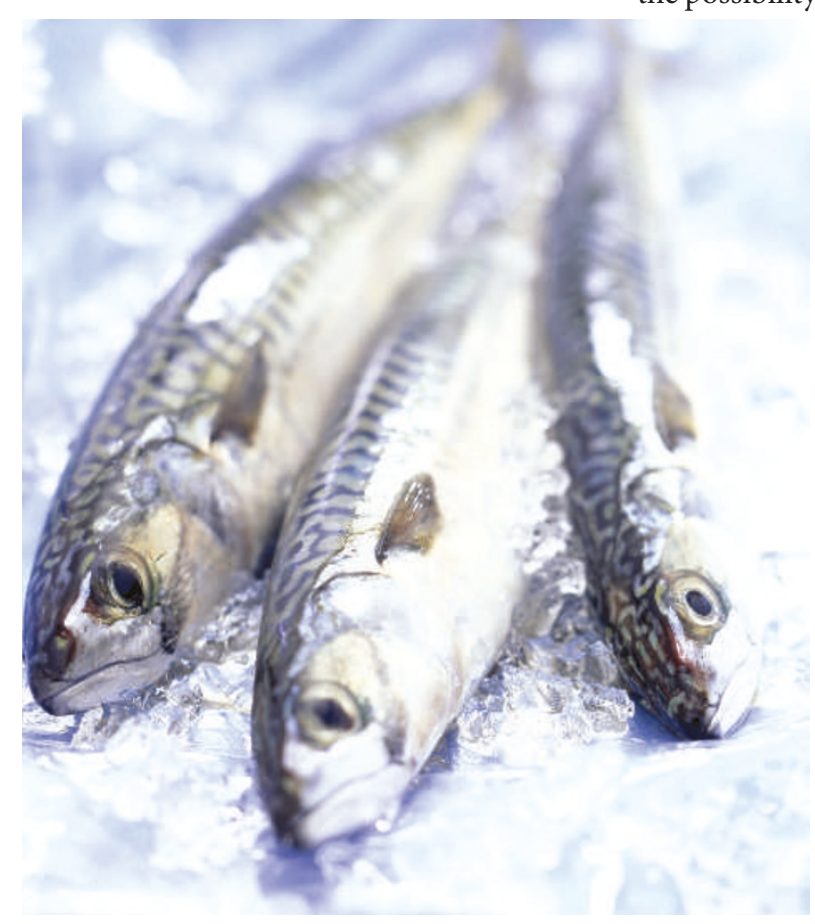

Something fishy: mackerel is a good source of omega-3s.

stroke. In addition, the oxidation of omega-3 fatty acids can increase the level of low-density lipoprotein, reduce glycaemic control in diabetics, and reduce immunocompetence. She could also have elaborated on the significance of another omega- 3 fatty acid, DPA, and on adverse health effects in infants and adults of lipid-soluble toxins (such as dioxins and polychlorinated biphenyl) and of heavy metals in fish oils. I also expected her to say more about the possibility of switching to new sources of omega-3 fatty acids, such as genetically modified oils, as a way of delivering more highly concentrated sources of omega- 3 fatty acids. The advent of algal sources of DHA provides one of the few terrestrial sources of this fatty acid in a concentrated form. All this has been made possible by new technologies and improved processing techniques that ensure the stability and preserve the integrity of these unstable fatty acids.

These omissions aside, however, Allport's book provides an interesting and comprehensive account of the history of omega-3 fatty acids. It not only provides a clearly reasoned case for the benefits of having more omega- 3 fatty acids in the diet, but also offers practical advice about how to add these fats to our diet. The book is also well organized, with a clear contents list, comprehensive notes, time lines and index to enable readers to quickly locate a required topic.

Asim K. Duttaroy is in the Department of

Nutrition, University of Oslo, PO Box 1046

Blindern, Oslo N-0316, Norway.

\section{Learning from nature}

\section{Biomimetics: Biologically Inspired \\ Technologies \\ edited by Yoseph Bar-Cohen \\ CRC Press: 2006.527 pp. $€ 79.99$}

\section{Robert W. Cahn}

One touch of nature makes the whole world kin -

That all with one consent praise new-born Though they are made and moulded of
.

And give to dust that is a little gilt things past,

More laud than gold o'er-dusted.

Yoseph Bar-Cohen's multi-author volume Biomimetics, devoted as it is to a plethora of 'new-born gauds', has examples of gold coated in a variable thickness of dust. In defiance of Ulysses' cynicism in Shakespeare's Troilus and Cressida, there is only a little shiny dust on display here, and I suspect that readers will quickly brush it aside.

One of the chapters begins: "The field of biomimetics encompasses a broad range of topics, generally based on the concept of 'learning from Nature' in areas of Materials Science and Engineering (MSE). This 'learning' may be through inspiration in design, function or a combination of both." Until I read this book, that was my impression of biomimetics too. However, only 5 of the 20 chapters are focused on aspects of MSE, particularly mechanical properties. One of these centres on 'muscles' made from electroactive polymers, and includes some irresistible pictures of a teenage girl arm-wrestling with a synthetic arm.

The rest of the book has a strong emphasis on robotics, both macroscopic and nanoscopic, no doubt prompted by the editor's attachment to the Jet Propulsion Laboratory in Pasadena, with its mission of space and planetary exploration. Synthetic eyes and associated electronics also receive extensive attention. There are two unusual but interesting chapters devoted to design and optimization procedures that imitate the processes of 
biological evolution. One of these chapters, on 'genetic algorithms', contains some intriguing examples of this procedure, one of which even incorporates an ingenious analogue of sexual reproduction. Another example involves the optimum locations of a group of post offices to serve a community as economically as possible, and another considers the dynamic balancing of a gas-turbine shaft. Generally, the book contains much control and optimization theory, some of it alarmingly mathematical. It should also be pointed out that most of the optimization procedures discussed make use of computer simulation, rather than physical modelling.

Some chapters include detailed examples of applications of the principles set forth, whereas others, no less stimulating, describe in depth the relevant features of nature but barely hint at specific applications, such as the chapter on defence and attack mechanisms in biology. Only one (very long) chapter, on the 'mechanization of cognition', seems to be 'gilded dust'; I cannot see that it contributes anything to the theme of the book.

Bar-Cohen himself wrote the substantial opening and closing chapters, and suggests the next stages of research. He also penned the chapter on synthetic muscle, one of his research specialities.

This book differs sharply from earlier treatments of biomimetics, such as R. McNeill Alexander's Animal Mechanics (Sidgwick \& Jackson, 1968). Stephen Mann's Biomimetic Materials Chemistry (VCH, 1996), which I reviewed here ten years ago (Nature 382, 684;
1996), focused on materials chemistry. An important subset of this theme is the chemistry and microstructure of high-strength composites, and also spider silk, which clearly exerts great fascination - there is also a chapter on silk in Bar-Cohen's book.

The focus on robotics and on optimization by evolution seems to be unique and constitutes the main claim of Bar-Cohen's volume to widespread attention. Another feature of the book is the extreme care he has taken to obtain the critical opinions of 66 experts, and their ministrations evidently contributed much to the book's clarity of presentation.

Robert W. Cahn is in the Department of Materials Science and Metallurgy,

University of Cambridge, Pembroke Street, Cambridge CB2 3QZ, UK.

\section{Burning Bush}

\section{An exhibition in Australia highlights the country's bushfires.}

\section{Colin Martin}

Disastrous bushfires, which occur regularly in Australia during the scorching summer months, have scarred the country's collective psyche as well as its landscape. From colonial painter William Strutt's panoramic canvases of bushfires, such as the Black Thursday fires in 1851, to today's television news coverage of widespread forest devastation and burnt-out buildings, images of bushfires abound.

'Fireworks: Tracing the Incendiary in Australian Art' is an exhibition of more than 50 contemporary and historic works that show the impact of bushfires on the country's art. It reveals their continuing potency in stimulating the creativity of Australian artists, who depict both their dramatic pyrotechnic effects and their tragic consequences.

The exhibition includes works by contemporary Aboriginal artists, whose ancestors used fire as a land-management tool over tens of thousands of years. Exploring Australia's east coast in 1770 , Captain James Cook described the land as "a continent of smoke" and noted that "we saw smoke by day or fires by night wherever we came". As well as using campfires, Aborigines regularly set fire to areas of woodland and grassland to regenerate plant food for themselves and the animals they hunted, to sustain a viable food chain.

Systematic 'fire-stick farming' released nutrients into the soil and fostered fireloving plant genera, such as Banksia and Hakea, which require intense heat to release their seed. By reducing the area of thickly timbered country and creating open grassy woodlands, fire-stick farming limited the spread of fire. Its decline following European

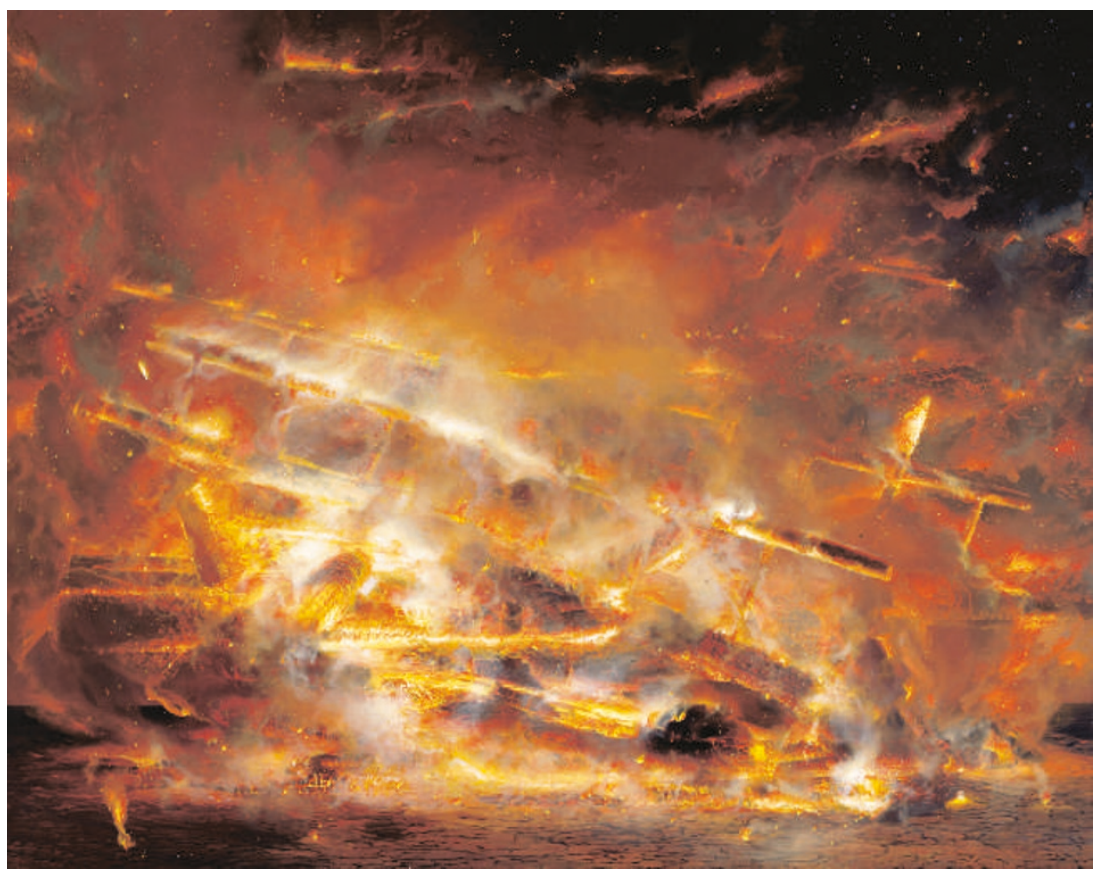

Flames of passion: Tim Storrier's The Ladder shows that fire stimulates the imagination.

settlement made it easier for bushfires to spread quickly in southeast Australia.

Many of the exhibited works show the immediate aftermath of bushfires, in charred, still-smouldering landscapes. Others show green undergrowth regenerating among blackened tree trunks, like a phoenix rising from the ashes, hinting at the redemptive power of fire in the Australian landscape. The most dramatic works, however, depict fire as the protagonist. In The Ladder by Tim Storrier (shown here), it dominates the foreground. The canvas is covered with shards of flaming light, swirling smoke and heat, across which a burning ladder has fallen to create a claustrophobic space from which we cannot escape.

The images in 'Fireworks' illuminate how Australian inhabitants have interacted with their environment, from Aborigines to the present-day population. Both scientists and artists, who observe the environment closely in order to depict it, are now encouraging a return to Aboriginal methods of land management to combat bushfires.

'Fireworks' can be seen at the University Art Museum in Brisbane, Queensland, from 19 December until 4 February 2007.

Colin Martin is a London-based writer. 